\title{
Non-TAVR Bailout for Acute Severe Aortic Regurgitation After Balloon Aortic Valvuloplatsy by Using Fogarty ${ }^{\circledR}$ Catheter
}

\author{
Ikki Komatsu, $\mathrm{MD}^{1 *}$, Hirotsugu Mitsuhashi, MD, PhD' ${ }^{1}$, Joji Ito, $\mathrm{MD}^{2}$ \\ ${ }^{1}$ Department of Cardiology, St. Luke's International Hospital, Heart Center, Tokyo, Japan \\ ${ }^{2}$ Department of Cardiovascular Surgery, St. Luke's International Hospital, Heart Center, Tokyo, Japan
}

\begin{abstract}
Acute severe aortic regurgitation (AR) after balloon aortic valvuloplasty (BAV), which rarely occurs, can cause catastrophic deterioration of a patient's hemodynamics. The emergent conversion of transcatheter aortic valve replacement (TAVR) or open heart surgery can be considered as bailout procedures for this situation; however, these are not ideal or safe options. We developed a novel bailout method for acute AR without TAVR or surgical conversion. The patient was an 82-year-old woman with severe aortic stenosis who had been treated for interstitial pneumonia for more than 10 years and in whom BAV was scheduled for worsening dyspnea. Through BAV performed using a retrograde approach with an $18-\mathrm{mm}$ balloon, we treated the entrapment of the non-coronary leaflet, the vital sign of which had deteriorated due to severe AR. We attempted to manipulate a pigtail catheter to push back the entrapped leaflet; however, the attempt failed. Next, we advanced a Fogarty catheter into the space between the wall of the sinus of Valsalva and the entrapped non-coronary leaflet with the aim of pushing it back. Inflating the balloon restored the leaflet movement, which successfully reduced $A R$ and obtained hemodynamic stability. This procedure successfully avoided further invasive bailout procedures.

Copyright $\odot 2018$ Science International Corp.
\end{abstract}

Fax +1 2037853346

E-Mail: jshd@scienceinternational.org

http://structuralheartdisease.org/

\author{
(C) 2018 Journal of Structural Heart Disease \\ Published by Science International Corp. \\ ISSN 2326-4004 \\ Accessible online at: \\ http://structuralheartdisease.org/
}

Key Words

Balloon aortic valvuloplasty - Complication - Acute aortic regurgitation $-B$ ailout procedure $\cdot$ Transcatheter

\section{Introduction}

Balloon aortic valvuloplasty (BAV) was the only interventional therapeutic option for patients suffering from severe aortic stenosis (AS) for whom open heart surgery is not indicated. With the emergence of transcatheter aortic valve replacement (TAVR) as an alternative treatment option for such situations, the indications for BAV as a bridging therapy for TAVR have expanded.

Acute aortic regurgitation (AR) is a rare complication of BAV that is reported as to occur in $0.8-1.5 \%$ of all procedures [1-2]. This complication is clinically important, as a sudden hemodynamic collapse can lead to a fatal outcome. However, the risk of AR is difficult to predict before BAV, and emergent actions are therefore required. The emergent conversion of TAVR or open heart surgery can be considered as bailout procedures for this situation [3-4]. However, these are not ideal or sufficiently safe, as they require additional manpower, preparation time, and availability of valve devices. Furthermore, it is obviously not adequate for institutions without experience to rapidly switch and

* Corresponding Author:

Ikki Komatsu, MD

Department of Cardiology

St. Luke's International Hospital, Heart Center

9-1 Akashicho, Chūō, Tokyo, 104-8560, Japan

Tel.: +81 33541 5151; Fax: +81 33541 5151; E-Mail: ikki2002@msn.com 

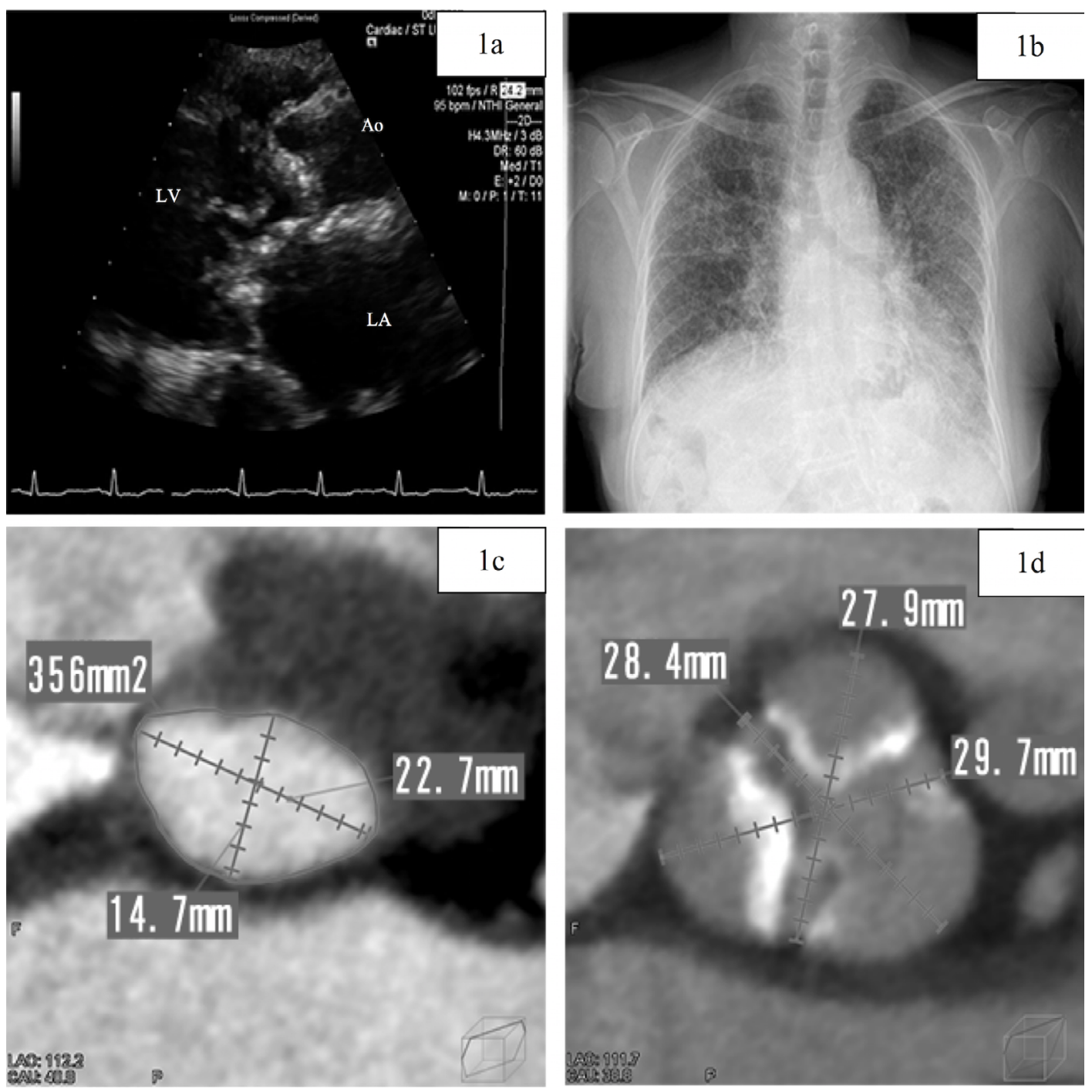

Figure 1. Periprocedural images. Panel A. Parasternal transthoracic echocardiogram image obtained in the systolic phase. Panel B. Chest X-ray. Axial computed tomography scan of the aortic annulus (Panel C) and sinus of Valsalva (Panel D). $A o=$ aorta; $L V=$ left ventricle; $L A=$ left atrium

prepare for TAVR [4]. Furthermore, poor outcomes have been reported in cases of emergent conversion to open heart surgery [5]. As such, there is need for a novel quick bailout technique that does not involve greater invasion.

We successfully treated acute AR without TAVR or surgical conversion. A Fogarty catheter was used to push back the entrapped aortic valve leaflet that was the cause of acute AR. This action reduced the severity of the AR from severe to mild, which successfully stabilized the patient's hemodynamics, thereby avoiding intubation or the need for additional TAVR or surgical conversion. We believe this procedure could reduce the need for emergent TAVR or surgical intervention and improve patient outcomes.

\section{Case Presentation}

An 82-year-old Japanese woman was referred to our valvular heart disease department due to a heart murmur and a newly developed worsening of dyspnea on effort. Transthoracic echocardiography (TTE) revealed severe aortic valve stenosis, septal bulge, and mild-moderate mitral stenosis. She had been 

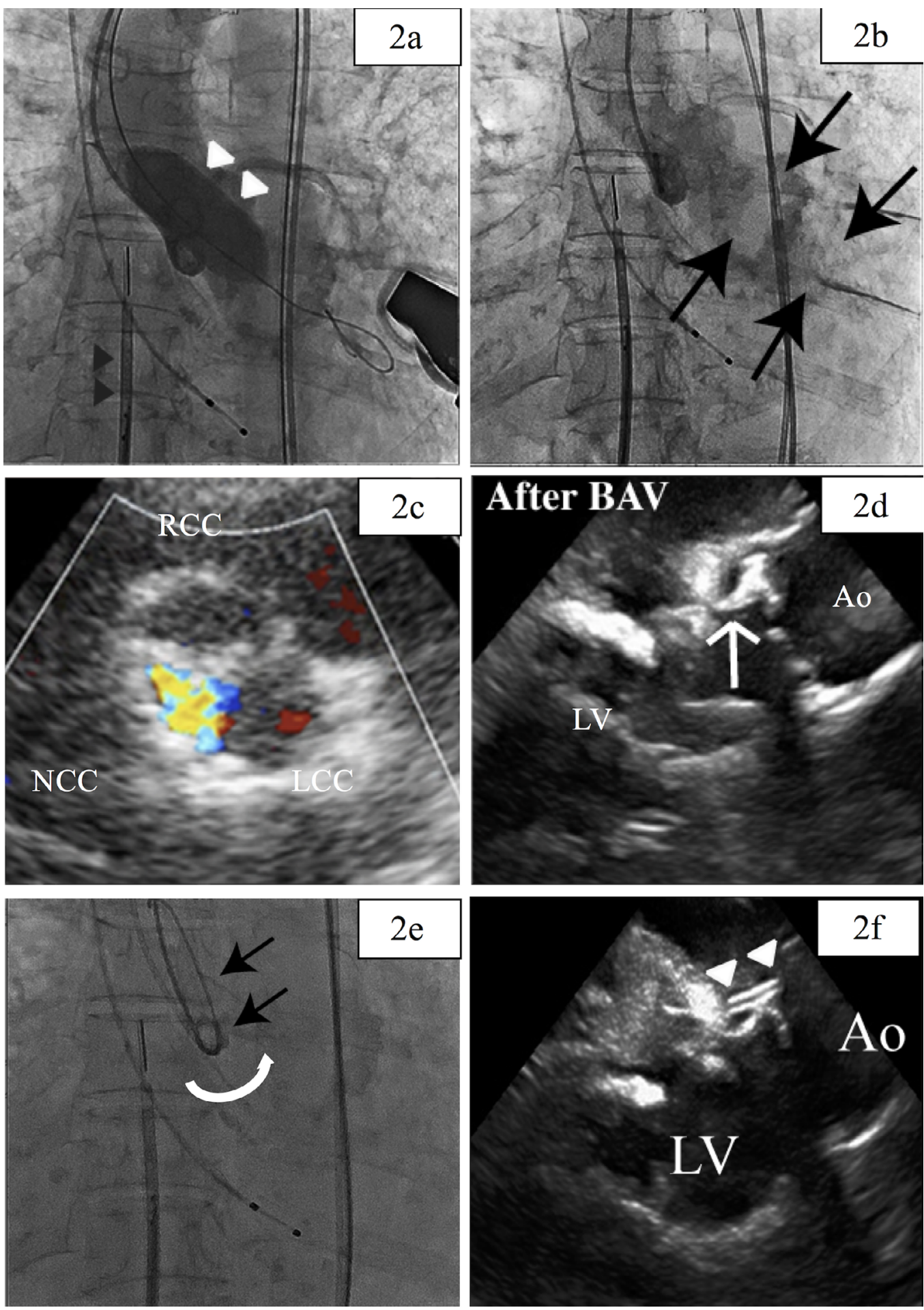

Figure 2. Balloon aortic valvuloplasty (BAV), acute aortic regurgitation (AR), and the bailout procedure with pigtail rotation. Panel A. BAV with an 18-mm balloon (white triangle). The black triangle indicates intracardiac echocardiography (ICE). Panel B. Acute severe AR after BAV (black arrow). Panel C. Transthoracic echocardiogram image of acute AR from the non-coronary cusp. Panel $D$. ICE image of the entrapped non-coronary leaflet (white arrow). Panel E. Rotating the pigtail catheter (white arrow) with a stiff wire (black arrow). Panel F. ICE image confirming the pigtail positioning at the entrapped non-coronary leaflet (white triangle). $\mathrm{RCC}=$ right coronary cusp; $\mathrm{LCC}=$ left coronary cusp; $\mathrm{NCC}=$ non-coronary cusp; $\mathrm{Ao}=$ aorta; $\mathrm{LV}=$ left ventricle. 

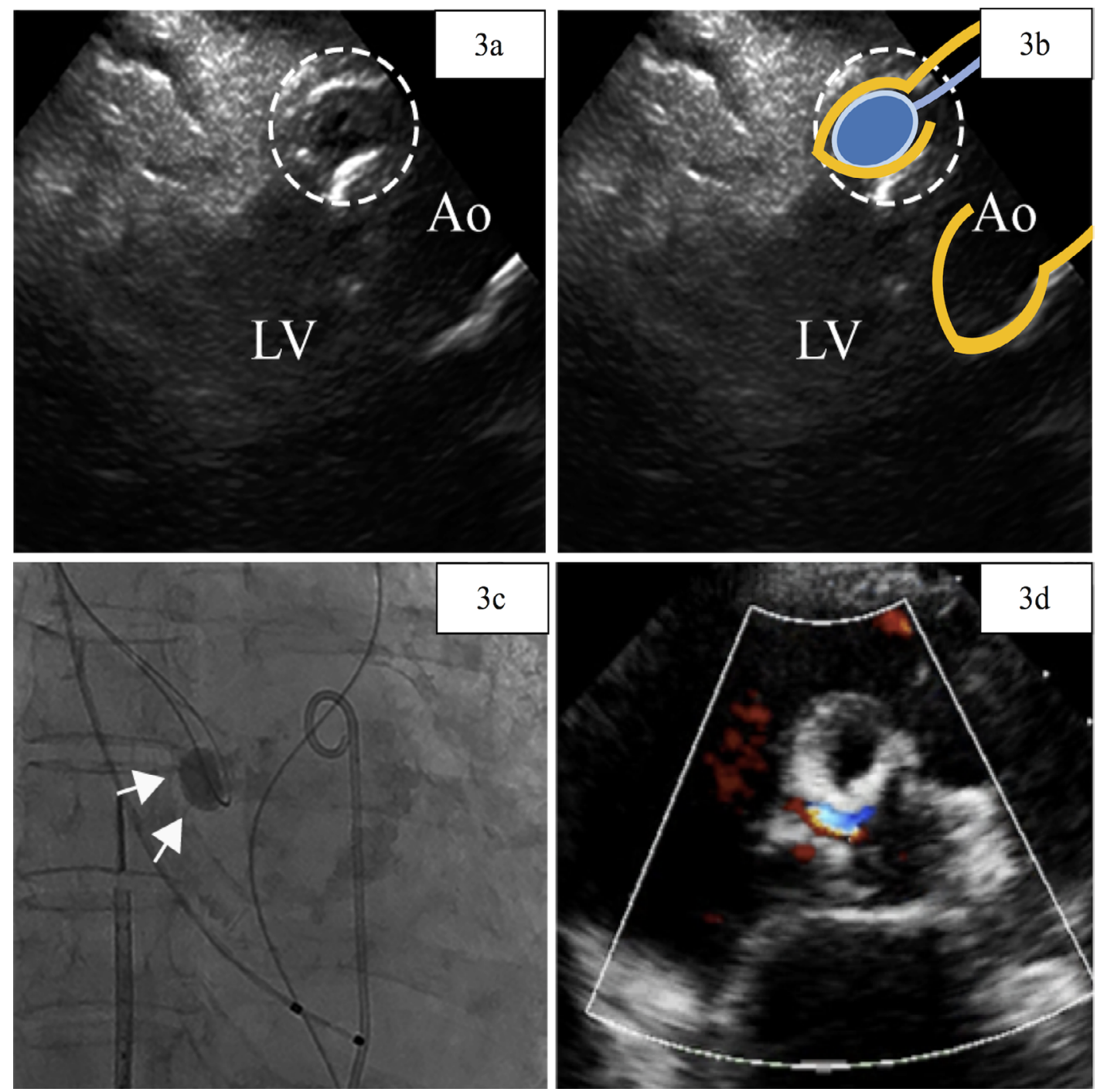

Figure 3. Intracardiac echocardiography image during inflation of the Fogarty catheter (Panel A) and a schematic image (Panel B). The blue circle indicates the Fogarty catheter. Panel C. Inflation of the Fogarty catheter (white arrow). Panel D. Transthoracic echocardiography image of aortic regurgitation reduction. Ao = aorta; LV = left ventricle.

treated for hypersensitivity pneumonitis and interstitial pneumonia for approximately 10 years with home oxygen therapy and oral steroids. Her STS score was $16.5 \%$. As she had both heart and lung problems, the cause of her symptoms was not clear. After discussion between the heart team and a pulmonologist, BAV was scheduled to confirm whether aortic valve intervention would reduce her symptoms. Preprocedural TTE revealed severe aortic stenosis with a $0.59 \mathrm{~cm}^{2}$ valve area (planimetry); peak velocity, $3.8 \mathrm{~m} / \mathrm{s}$; mean pressure gradient, $37.2 \mathrm{mmHg}$; and max pressure gra-

Journal of Structural Heart Disease, February 2018 dient, $59.0 \mathrm{mmHg}$. No AR, mild-moderate mitral stenosis, or septal bulge were observed. (Figure 1A)

Chest X-ray and lung computed tomography (CT) showed a reticular shadow pattern (Figure 1B). The annular area calculated on cardiac CT was $356 \mathrm{~mm}^{2}$, with a diameter of $24.6 \times 19.7 \mathrm{~mm}$ (Figure 1C and 1D).

The BAV procedure was performed under local anesthesia with light sedation controlled by an anesthesiologist. A temporary pacemaker was inserted via the right jugular vein, an 11-F sheath in the left femoral artery, a 5-F sheath in the right femoral artery with a $5-\mathrm{F}$ pigtail catheter seated in the non-coronary 


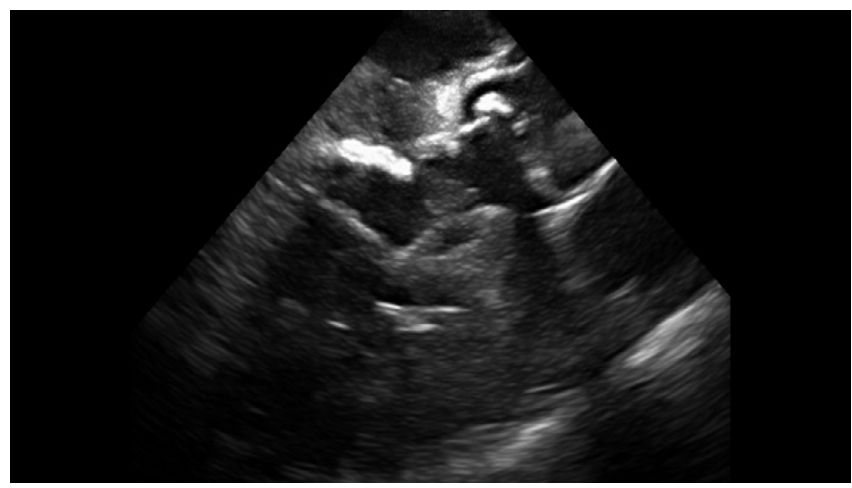

Video 1. Intracardiac echocardiography showing the entrapped non-coronary leaflet. Motion loss and entrapment of the non-coronary leaflet are observed. View supplemental video at https://doi.org/10.12945/j.jshd.2018.038.17.vid.01.

cusp, and a 10-F sheath in the right femoral vein with intracardiac echocardiography (ICE; ViewFlex, St. Jude Medical Japan Co., Ltd, Tokyo, Japan). Heparin was administered with a target activated clotting time of 200-300 s. The contrast media was diluted twice with saline.

The aortic valve was crossed with a 5-F AL1.0 diagnostic catheter (Terumo Medical Corporation, Tokyo, Japan) using a 0.035" Radiforcus straight wire (Terumo Medical Corporation), which was exchanged for a manually shaped Amplatz Super Stiff Wire (Boston Scientific Corporation, Tokyo, Japan). An 18-mm NuMed balloon (Trytech Co., Tokyo, Japan) was inserted into the aortic valve and inflated with rapid pacing (Figure 2A).

Immediately after ballooning, the patient's systolic blood pressure dropped to $60 \mathrm{mmHg}$, and she complained of chest discomfort. Aortic root angiography revealed severe AR (Figure $2 \mathrm{~B}$ ). ICE and TTE confirmed movement loss and entrapment of the non-coronary leaflet. AR color jet was mainly observed in this area (Figure 2C and 2D and Video 1). The pigtail catheter was inserted into the left ventricle without any resistance; this is referred to as the "free floating pig tail sign". The aortic-left ventricle pressure gradient was almost equivalent after BAV due to entrapment of the non-coronary leaflet. TTE and ICE revealed no pericardial effusion.

We were able to control the patient's blood pressure with catecholamine, and thus she did not require mechanical support. However, because her $\mathrm{SpO}_{2}$ lev-

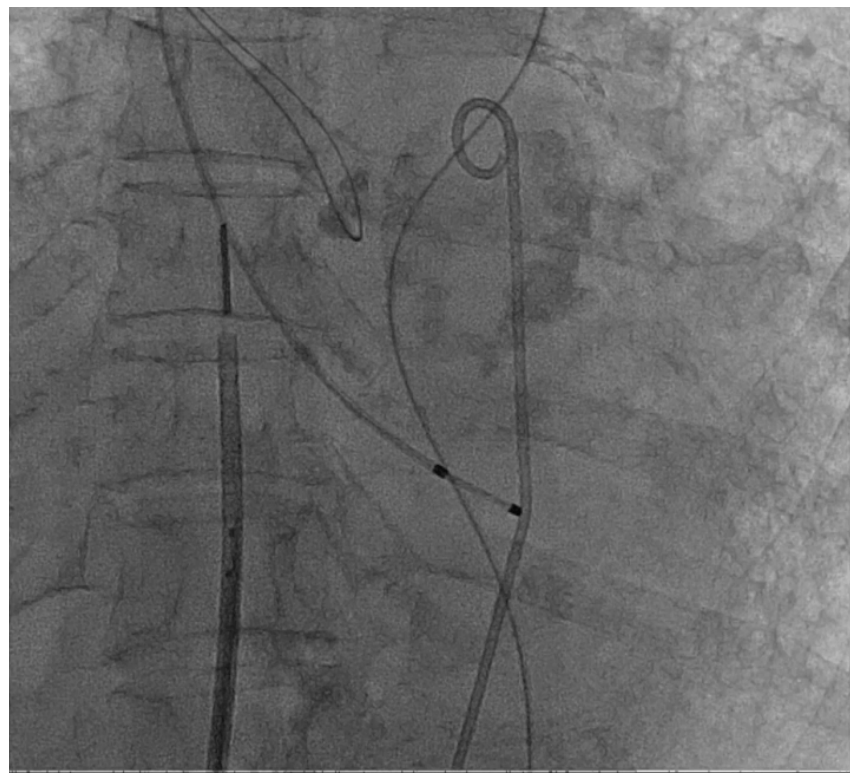

Video 2. Cineangiography showing inflation of the Fogarty catheter. View supplemental video at https://doi.org/10.12945/j. jshd.2018.038.17.vid.02.

el dropped and central venous pressure increased, we added noninvasive positive pressure ventilation (NPPV).

Our patient was considered unsuitable for emergency TAVR or surgical conversion for several reasons. First, TAVR was not clearly indicated, as we had not yet determined whether AS would be effective for improving the patient's symptoms. Second, as our institution had limited experience with TAVR at that time, we thought that the emergent preparation of TAVR would have the potential to cause further problems. Finally, the patient had a high STS score and lung disease, and surgical intervention was considered to be too risky. Thus, we decided bailout was required without conversion to TAVR or open surgery.

We first attempted mechanical push back using the pigtail catheter. We ensured insertion of the catheter between the entrapped non-coronary leaflet and the wall of the sinus of Valsalva by ICE and then rotated it repeatedly. However, we encountered huge resistance, with no movement of the non-coronary leaflet. We then tried to use a stiff wire in the pigtail to stiffen the catheter itself; however, this was not effective (Figure 2E and 2F).

We needed another option to push the non-coronary leaflet back from its entrapped position. We 
attempted to use a Fogarty catheter (Edwards Lifesciences Corporation, Tokyo, Japan) because we thought that a sphere-shaped balloon would fit in the shape of the sinus of Valsalva and the coronary cusp and that it could be advanced into the narrowed space between the entrapped coronary leaflet and the wall of the sinus of Valsalva using an over-thewire lumen.

A 4-F compatible, 9.0-mm balloon expansion size Fogarty catheter was selected. The $0.035^{\prime \prime}$ wire was switched to a $0.025^{\prime \prime}$ wire as the balloon was compatible with a $0.025^{\prime \prime}$ wire. Tracking with the wire, the Fogarty catheter was successfully advanced and inserted into the slit of the non-coronary leaflet and the wall of the sinus of Valsalva, which was confirmed by ICE and fluoroscopy. The balloon was gently inflated three times for $\sim 10$ s each time (Video 2). After the inflation, TTE and ICE showed that non-coronary leaflet movement had been restored and that the degree of AR was reduced to mild. Findings of aortic root angiography were also compatible with $A R$ reduction. The patient was transferred to the intensive care unit without NPPV or catecholamine support.

On postoperative day 1 , the patient's respiratory condition worsened, and she required NPPV again; however, right and left heart diagnostic catheterization on postoperative day 2 revealed no increases in pulmonary capillary wedge pressure or left ventricular end-diastolic pressure, which ruled out heart failure with AR. This was therefore diagnosed as an acute worsening of lung disease. The patient gradually recovered and was discharged on postoperative day 15 without further complications. Follow-up TTE showed mild AR; moderate AS with $0.8 \mathrm{~cm}^{2}$ (planimetry); peak velocity, $3.3 \mathrm{~m} / \mathrm{s}$; mean pressure gradient, $26.2 \mathrm{mmHg}$; and max pressure gradient, $43.9 \mathrm{mmHg}$.

\section{Discussion}

To our knowledge, this is the first case report describing the successful bailout of acute AR after BAV using a Fogarty catheter. Postmortem images of acute AR due to leaflet entrapment after BAV were previously shown by Treasure et al. [7], who reported that malalignment of the irregular surfaces of the fractured calcific nodule entrapped the non-coronary leaflet in an open position and mechanically hindered the ability of the leaflet to close. Hara et al. reported that BAV created intraleaflet fractures within the aortic valve calcified deposit [8], and Mizuno et al. reported a case in which cracks made by BAV were observed in the chunky calcification at the coronary leaflet on postmortem images 6 months after BAV [9]. Based on these reports, we hypothesize that the entrapment in our case might have been caused by the inside-bending of cracked calcified nodular deposits, which locked the leaflet into an open position. ICE in this case clearly showed bending of the non-coronary leaflet.

The Fogarty catheter was suitable for our requirements in the present case for two reasons. First, as a sphere-shaped balloon was required to fit the shape of the wall of the sinus of Valsalva and the coronary cusp, a coronary or endovascular over-the-wire type balloon would not have been appropriate due to their rectangular shape. Second, it was considered difficult to advance the balloon into position accurately; however, this was a necessary aspect of the bailout procedure. An over-the-wire type balloon met these requirements, as this type of balloon can be tracked by the wire and manipulated and inserted into the slit of the entrapped leaflet and the wall of the sinus of Valsalva using a MultiPurpose or Judkins Right catheter, with echocardiography performed as a backup.

We believe that our method can decrease the need for emergency TAVR or surgical conversion, which may decrease further complications of acute AR after BAV. It might be possible to use this Fogarty catheter bailout technique to stabilize a patient's hemodynamics during preparation for TAVR or surgical conversion.

In conclusion, the Fogarty catheter can be safely used for bailout of acute AR caused by an entrapped coronary leaflet after BAV. We believe that using this technique as a bailout procedure can reduce the need for emergency TAVR conversion or surgery.

\section{Acknowledgments}

We thank Drs. Osamu Okada and Kohei Abe, who were involved in the treatment of the patient. 


\section{Conflict of Interest}

The authors have no conflict of interest relevant to this publication.

\section{Comment on this Article or Ask a Question}

\section{References}

1. Loncar S, Perlman G, Planer D, Meroz Y, Gilon D, Lotan C, et al. Acute aortic regurgitation and hemodynamic collapse after balloon aortic valvuloplasty. Int J Cardiol. 2014;171:88-89. DOI: 10.1016/j.ijcard.2013.11.063

2. Ben-Dor I, Pichard AD, Satler LF, Goldstein SA, Syed Al, Gaglia MA Jr, et al. Complications and outcome of balloon aortic valvuloplasty in high-risk or inoperable patients. J Am Coll Cardiol Intv. 2010;3:1150-1156. DOI: 10.1016/j.jcin.2010.08.014

3. Seiffert M, Conradi $L$, Baldus $S$, Schirmer J, Blankenberg S, Reichenspurner $\mathrm{H}$, et al. Severe intraprocedural complications after transcatheter aortic valve implantation: Calling for a heart team approach. Eur J Cardiothorac Surg. 2013;44:478-484. DOI: 10.1093/ejcts/ezt032

4. Gurvitch R, Tay EL, Wijesinghe N, Ye J, Nietlispach F, Wood DA, et al. Transcatheter aortic valve implantation: Lessons from the learning curve of the first 270 highrisk patients. Catheter Cardiovasc Interv. 2011;78:977-984. DOI: 10.1002/ccd.22961

5. Hein R, Abdel-Wahab $M$, Sievert $H$, Kuck $\mathrm{KH}$, Voehringer $M$, Hambrecht $R$, et al. Outcome of patients after emergency conversion from transcatheter aortic valve implantation to surgery. Eurolntervention. 2013;9:446-451. DOI: 10.4244/EIJV914A73

6. Dean LS, Chander JW, Saenz CB, Baxley WA, Bulle, TM. Severe aortic regurgitation complicating percutaneous aortic valve valvoloplasty. Cathet Cardiovasc Diagn. 1989;16:130-132. PMID: 2914318

7. Tresure CB, Schoen JS, Treseler PA, Bital JA. Leaflet entrapment causing Acute Severe Aortic Insufficiency During Balloon Aortic Valvuloplasty Clini Cardiol. 1989;12:405408. PMID: 2743630

8. Hara H, Pedersen WR, Ladich E, Mooney
M, Virmani R, Nakamura $M$, et al. Percutaneous balloon aortic valvuloplasty revisited: Time for a renaissance? Circulation. 2007;115:e334-e348. DOI: 10.1161/CIRCULATIONAHA.106.657098

9. Mizuno A, Kawazoe K, Niwa K. Efficacy of percutaneous balloon aortic valvuloplasty visualized on computed tomography. Circ J. 2014;78:1002-1003. PMID: 24317110

Cite this article as: Komatsu I, Mitsuhashi $\mathrm{H}$, Ito J. Non-TAVR Bailout for Acute Severe Aortic Regurgitation After Balloon Aortic Valvuloplatsy by Using Fogarty ${ }^{\circledast}$ Catheter. Structural Heart Disease. 2018;4(1):21-27. DOI: https://doi. org/10.12945/j.jshd.2018.038.17 